\title{
Investigation and application of the frustrated-total-internal-reflection phenomenon in optical fibers
}

\author{
Kambiz Rahnavardy, Vivek Arya, Anbo Wang, and Joseph M. Weiss
}

\begin{abstract}
A detailed investigation of the frustrated-total-internal-reflection (FTIR) phenomenon in silica-glassbased optical fibers and its application to simple intensity-modulated strain and pressure sensors is presented. Such sensors may be readily fabricated with silica-based fibers and can be easily modified with sapphire fibers for high-temperature industrial applications where conventional silica-based fiber sensors are not feasible. We present the all-fiber FTIR sensor and show good correlation between theory and experiment. We also present results for the design and implementation of a prototype FTIR-based fiber pressure sensor. (C) 1997 Optical Society of America
\end{abstract}

Key words: Frustrated total internal reflection, fiber optics, sensors.

\section{Introduction}

Optical-fiber-based sensors are classified as extrinsic and intrinsic, which refers to the sensing region's being outside the fiber or inside the fiber, respectively. In intrinsic sensors the optical waves in the fiber are affected directly by the incident perturbation (the parameter being measured), and changes in the output intensity give an indication of the magnitude of the disturbance. Such intrinsic fiber sensors include the sapphire fiber-based Fabry-Perot interferometric sensor, 1,2 which has been successfully implemented for high-temperature measurements. In extrinsic sensors the fiber serves only to carry optical power to, and the sensing information from, an external region. Such sensors are often immune to vibration and temperature fluctuations. Extrinsic sensors based on the extrinsic Fabry-Perot interferometric scheme have been implemented with circular core single-mode 3 and elliptical core twomode optical fibers for the measurement of strain,

K. Rahnavardy, V. Arya, and A. Wang are with the Fiber \& Electro-Optics Research Center, Bradley Department of Electrical Engineering, Virginia Polytechnic Institute and State University, Blacksburg, Virginia 24061-0356. J. M. Weiss is with the Fossil Plant Operations Program, Electric Power Research Institute, 3412 Hillview Ave., Palo Alto, California 94304-1395.

Received 9 May 1996; revised manuscript received 7 July 1996. 0003-6935/97/102183-05\$10.00/0

(C) 1997 Optical Society of America temperature, ${ }^{4}$ and vibrational mode analysis in smart structures. ${ }^{5}$

Fiber sensors are also classified according to the transduction mechanism, which brings about a change in some property-intensity, phase, polarization, modal content, and so forth-of optical waves propagating in the fiber. Hence the corresponding sensors are known as intensity based, phase based or interferometric based, polarimetric type, and modal content based. Intensity-based fiber sensors offer the advantages of ease of fabrication, robustness, and simplicity of signal processing. Frustrated totalinternal reflection (FTIR) is one such transduction mechanism and is investigated here for the implementation of simple intensity-based optical fiber sensors.

When light is incident from a higher index of refraction material toward a lower index of refraction material, there is total internal reflection when the angle of incidence exceeds the critical angle. Although no power is transferred to the second medium, an evanescent field exists in it. If there is a boundary to a third medium optically denser than the second medium, and if the thickness of the second medium is insufficient for the evanescent field to decay, then the total internal reflection is frustrated and power is coupled from the first medium to the third medium. This phenomenon is known as FTIR and is analogous to the quantum-mechanical tunneling of the electrons across narrow potential barriers. ${ }^{6,7}$

Two general approaches have been investigated to 
use FTIR as a transduction mechanism in optical fiber sensors. The first approach is to use the evanescent field produced at the end of a properly polished optical fiber. This was demonstrated by Spillman and McMahon. ${ }^{7}$ They fabricated a hydrophone with an all-fiber FTIR sensor in which a narrow variable air gap separated two angular-polished optical fibers (lead-in and target fibers). Although they obtained reasonable sensitivity, their results were different from theoretical predictions. Palmer et al. proposed a different version of the sensor with a glass plate instead of the second fiber (target fiber) to solve the practical problems in the previous model. ${ }^{8}$ Following this idea, Grattan et al. constructed a pressure sensor. 9 Their experimental prototype yielded results close to theoretical prediction. The drawback in their work was the use of a relatively complex fixture that is not very practical when hightemperature applications are considered. Other groups proposed using the change in the refractive index of the intermediary media instead of the change in the length of the gap as the variable parameter. ${ }^{10}$ The second approach that has been proposed and demonstrated is use of the exponentially decaying transverse fields that are produced outside the core of the optical fiber. In some cases it is necessary to remove part of the fiber's cladding to have better access to these fields. ${ }^{11,12}$

Our purpose in this paper is to investigate the allfiber FTIR sensor theoretically and use it to implement simple and practical intensity-modulated strain and pressure sensors. In all these applications the objective is to minimize the number of elements in the sensor to make the sensor an attractive candidate for future modifications, which include high-temperature or pressure-sensing applications.

\section{Theory}

As was mentioned in the introductory section, FTIR is similar to the quantum-mechanical tunneling of electrons across narrow potential barriers and results when two dielectric media sandwich a sufficiently narrow strip of a third, optically less-dense, medium. If the three dielectric media are glass, air, and glass, the resultant transmission $T$ of the glassair-glass interface may be expressed $a^{13}$

$$
T=1-\left|\left(z^{2}+\delta^{2}\right)^{2}\left[\left(z^{2}-\delta^{2}\right)^{2}+4 z^{2} \delta^{2} \operatorname{coth}(\beta / 2)\right]^{-1}\right|,
$$

where

$$
\beta=(4 \pi x / \lambda) \sqrt{\left(n^{2} \sin ^{2} \theta-1\right)},
$$

and

$$
z=1 /(n \cos \theta), \quad \delta=-1 / \sqrt{\left(n^{2} \sin ^{2} \theta-1\right)},
$$

for light with its polarization perpendicular to the plane of incidence ( $s$ component). For light polarized in the plane of incidence ( $p$ component),

$$
z=\cos \theta / n, \quad \delta=\sqrt{\left(n^{2} \sin ^{2} \theta-1\right)} .
$$

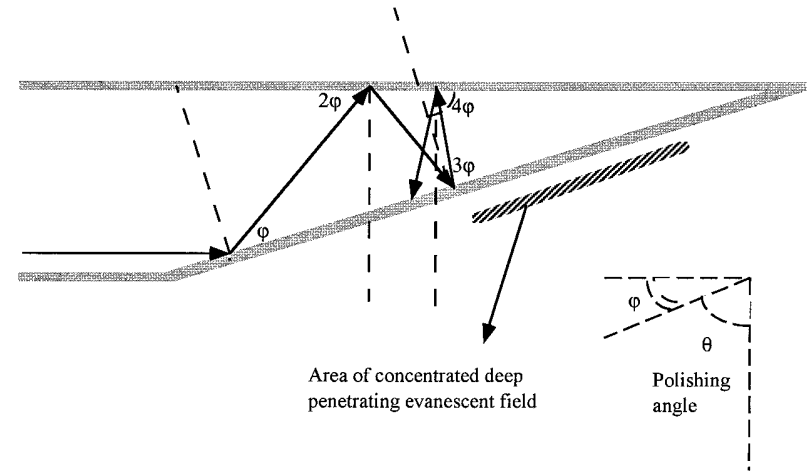

Fig. 1. Multiple reflections of the rays at a dielectric slab's angular-polished end.

Here $n$ refers to the refractive index of the glass material, $x$ is the gap distance between the two dielectric media, and $\theta$ is the angle of incidence. The reflectivity is complementary to the transmission when the possible scattering and absorption loss at the interface is negligible. It is seen that the optical power transmitted through the interface is extremely sensitive to small changes in the air gap. When the air gap vanishes, the incident power is transmitted through the interface without loss, but there is virtually no transmission when the air gap is more than a certain value dictated by FTIR. Moreover, because of the polarization dependence, there is an inherent self-calibrating property in FTIR that can be important in practical applications.

It is important to note two points. First, the light transmitted through the fiber cannot be represented by a plane wave, so the above equations cannot be directly used for FTIR-based fiber devices. These equations do, however, give a reasonable first-degree approximation for multimode fibers. More accurate results can be obtained with the ray optics approach and by considering the incident light as an average of rays with different directions distributed evenly inside a conical surface defined by the fiber's numerical aperture. For each ray, Eq. (1) is used to calculate the coupled power. The sum of the optical power carried by the rays approaches the actual amount of power coupled into the target fiber as the number of the modes becomes infinitely large (assuming a uniform modal power distribution). For beams that are incident upon a surface wider than their beam sizes, the approach is different because the incident beam must be decomposed into a sum of plane waves with different directions by means of surface Fourier transformation, with each component having a different FTIR-induced phase change.

The second point to note is that each ray in the lead-in fiber is subjected to multiple reflections before it loses its amplitude, scatters, or is reflected back. Figure 1 is a schematic of these multiple-reflection trajectories. It may also be noted (and proven analytically) that, when the rays are incident upon the polished tip of a dielectric slab, the consecutive angles of incidence may be represented as an arithmetic 


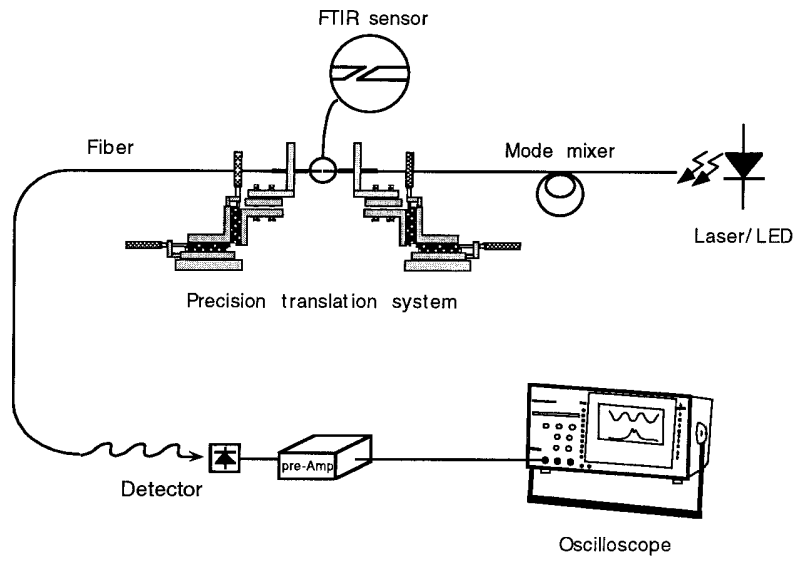

Fig. 2. Basic structure of a fiber-based FTIR sensor setup.

sequence. The polishing angle can then be chosen so that the second incidence on the endface is also totally reflected. Notice that for simplicity this diagram is based on a dielectric slab, but still it gives a good approximation of the meridional rays' behavior in a cylindrical structure such as an optical fiber.

When the fiber is polished at an angle such that the total internal reflection occurs only in the first incidence of the rays on the fiber's endface and when, for any reason, the target fiber's polished tip misses to interact with the produced evanescent field, it is virtually impossible to observe the FTIR phenomenon. But if the polishing angle is chosen to have the rays totally reflected off the polished tip for a second time, there is a much better chance to couple the power into the target fiber by means of FTIR. Moreover, because the light tends to become concentrated in a relatively small area of the polished tip, less modal noise and fewer misalignment problems are encountered.

\section{Experiment}

The basic setup of the FTIR experiment is shown in Fig. 2. A coherent light source was coupled with a connector into a multimode $84 / 125 \mu \mathrm{m}$ silica fiber. Future expansion of the current work can be accomplished without major modifications to include very high-temperature environments by replacing silica fiber with sapphire. This is because the fiber-opticbased FTIR sensor is intensity based, and mode coupling is not a concern. The source can be of any wavelength of interest as long as the roughness of the polished tip of the lead-in fiber is less than the operating wavelength. As an approximation, the roughness of the polished tip was kept below half of the wavelength used. This was to make sure that the FTIR phenomenon was kept in observable range. The obtained results demonstrate that this criterion is a practical estimate. It is clear that longer wavelengths can greatly reduce the problems of poorquality polishing and alignment. Wilner and Murarka have used $\mathrm{CO}_{2}$ lasers at $10.6 \mu \mathrm{m}$ in their proposed FTIR switch. ${ }^{14}$ However, we were bound to wavelengths below $1500 \mathrm{~nm}$ because of volume restrictions on the laser module and other practical considerations.

The source can be a light-emitting diode (LED) or any laser source. An LED is recommended in practical cases to avoid modal noise, although FTIR sensors are not affected critically by this type of noise. The reason is that FTIR-based sensors are intensity based, unlike the interferometric or phase-modulated sensors in which the basic interference signal can be severely affected. Moreover, because of the concentrating property of the angular polished tip, the speckle pattern that is created by launching a narrow band light into a multimode fiber is squeezed in a very small proportion at the sharp edge of the tip. Hence lateral misalignment is not critical.

In our experiment, the ends of the lead-in and target fibers were polished to a roughness of less than $0.3 \mu \mathrm{m}$ using a Logitech precision polishing machine CP30. With our criterion wavelengths of more than $600 \mathrm{~nm}$ could be used in the experiment. The polishing angle was the same for both fibers. The steeper the polishing angle, the less the created evanescent field will penetrate into the ambient media and hence the second fiber. In this case a sharp and distinct decrease in the power coupled into the second fiber, in response to normal gap separation of the two fibers, should be observed. However, the displacement of the two fibers in most practical cases will be confined along the fibers' axes. As a result, a steeper angle means less normal gap separation change for a certain longitudinal displacement length. According to the configuration of a given FTIR-based sensor, this trade-off should be properly balanced, and it is not always true that a steeper angle results in higher sensitivity. Other concerns, such as the use of micropositioners with limited resolution and the sensitivity to longitudinal and transverse vibrations, are also important in this trade-off.

To find the optimum polishing angle, we considered the area beneath the transmission $T$ versus gap displacement curve as a measure of the sensor's sensitivity. The sensitivity of the sensor is inversely proportional to this area. In Fig. 3 the reciprocal of this area is plotted as a function of the polishing angle for two different polarization states and wavelengths. As the curves show, a $76^{\circ}$ fiber tip will result in a very good sensitivity for $p$ polarization in both wavelengths. Because the secondary evanescent field creation is of utmost importance, we selected this polishing angle so that the secondary incident rays are also subject to total internal reflection. (With regard to Fig. 1, the incidence angle for the secondary reflection in this case is $48^{\circ}$.) The power coupled into the second fiber is detected as a micrometertranslator stage moves the target fiber away from the lead-in fiber in certain longitudinal intervals. The results are shown in Figs. 4 and 5.

\section{Results and Discussion}

The experimental results in Figs. 4 and 5 are shown along with the theoretical predictions. The theoretical curves are first-order approximations that con- 


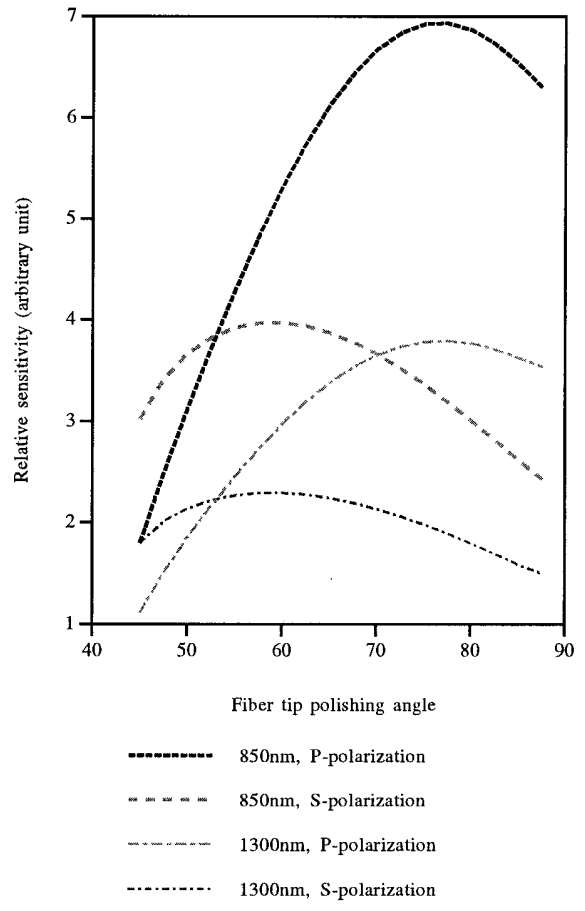

Fig. 3. FTIR sensor sensitivity indicator.

sider only the meridional rays with plane-wave calculations [Eqs. (1)-(4)]. The curves show clearly that the secondary rays and the evanescent field they produce play the dominant role in coupling the light through the target fiber; the experimental results at both wavelengths closely fit the curve for the secondary FTIR effect. The reason for the close fit is clearer if we consider the imperfections in the flatness of the fiber tip as well as its surface quality. It is plausible to assume that only a fraction of the polished surface is elevated enough to be in the effective range of the produced evanescent fields. The points of incidence for the primary rays that go through total internal

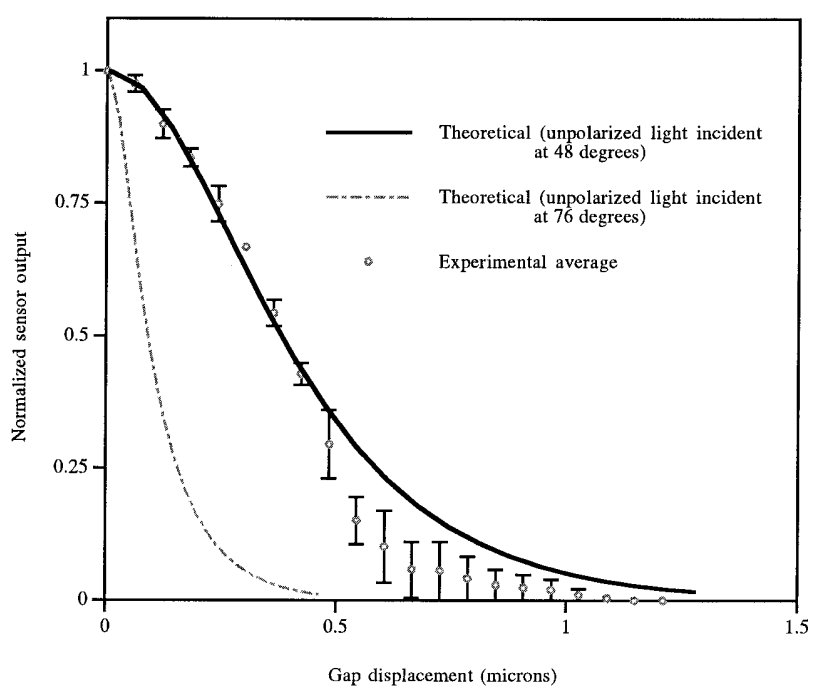

Fig. 4. Theoretical and experimental values for the FTIR sensor at $1300 \mathrm{~nm}$.

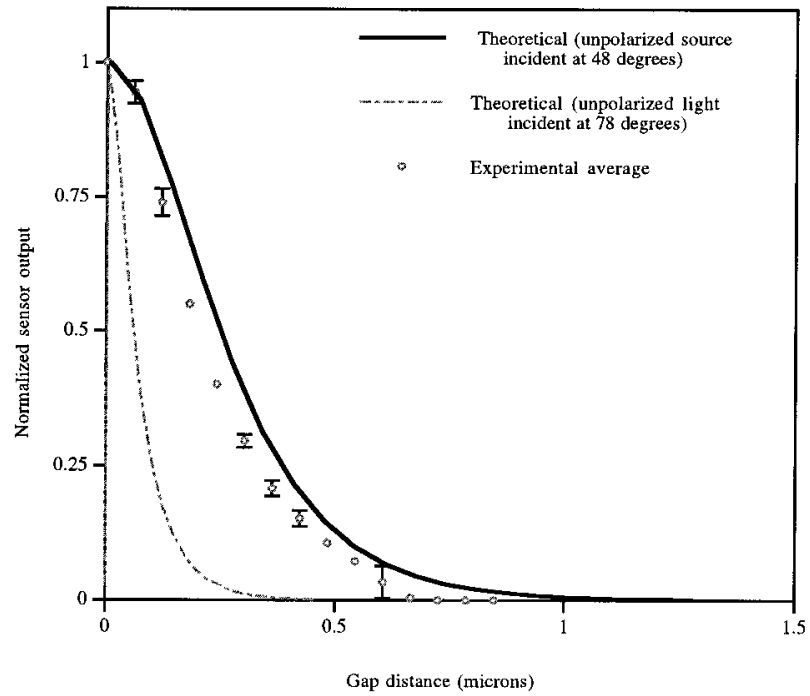

Fig. 5. Theoretical and experimental values for the FTIR sensor at $850 \mathrm{~nm}$.

reflection are distributed evenly across the polished surface. Therefore only a very small fraction of the primary rays can be captured through the FTIR effect. The situation is different for the secondary rays. As was mentioned before, the secondary rays are squeezed in a relatively small portion of the tip's surface, and their fields penetrate more into the air gap and the second medium. As a result, by proper alignment of the tips, we are able to couple the power into the second fiber with very low loss. In our experiment we have obtained initial alignments with losses of less than $0.3 \mathrm{~dB}$.

The most critical problem in this experiment is the attraction between the two delicately polished tips. Because of the small dimensions and negligible weight, the fiber tips act as springs. Consequently, when the two tips are in the vicinity of each other, they exert a strong attractive force on each other, which results in abrupt switching changes in the output power. To solve this problem partially in our case, the structure shown in Fig. 6 was built. The two fibers were covered by capillary metallic tubes right to their tips to reduce the spring effect and also to keep them in firm and easy-to-align shape. The diameter of the capillary tubes was slightly larger than the diameter of the fibers, and the remaining space was filled with epoxy. The output power was then monitored.

This prototype was tested to measure the vacuum pressure inside a plastic tube. The pressure inside the sensor's cavity was the room pressure. The gap between the fibers increased when the ambient pressure dropped below the room pressure as the cavity began to expand. This is detected by the built-in FTIR sensor. The result is shown in Fig. 7. It is obvious that with the configuration of our prototype sensor the achieved sensitivity is relatively low compared with the potential dynamic range that we theoretically anticipated. However, the result shows 


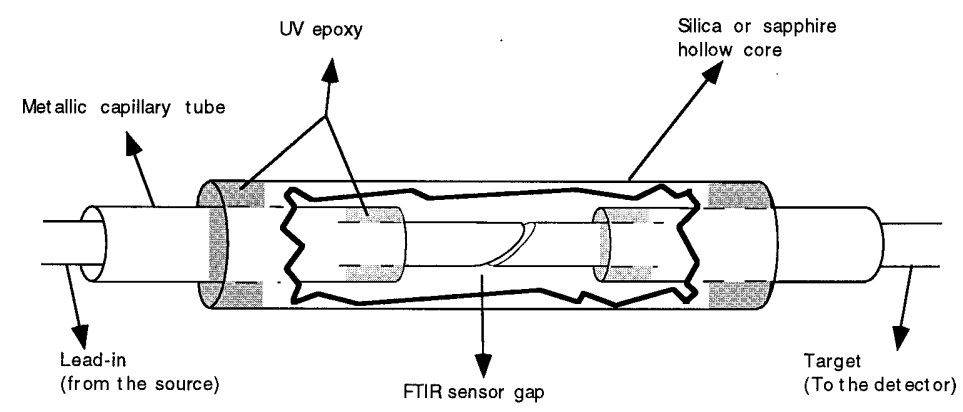

Fig. 6. Schematic of a fiber-based FTIR pressure sensor.

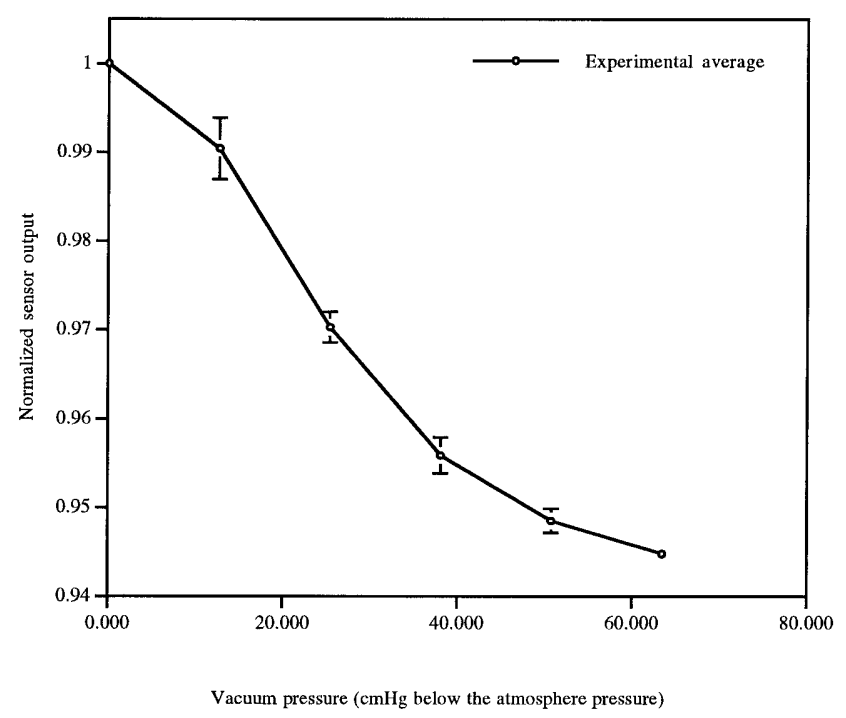

Fig. 7. Output of the FTIR sensor versus vacuum pressure.

the possibility of maintaining the alignment of the sensor and the practical use of it. It is feasible to modify this sensor to make it single ended, which makes it more practical.

\section{Conclusion}

We have presented a systematic evaluation of the FTIR phenomenon in silica-based optical fibers. The basic theory behind sensors based on the FTIR effect was compared with the experimental results. A close correlation was observed between the theory and the experiments, which is better than previous work in the same category. A prototype with a minimum number of components was fabricated to measure pressure changes inside a tube and was tested successfully.

The investigation above is part of a research grant on high-temperature optical-fiber sensors funded jointly by the National Science Foundation and the Electric Power Research Institute.

\section{References}

1. A. Wang, S. Gollapudi, K. A. Murphy, R. G. May, and R. O. Claus, "Sapphire-fiber-based intrinsic Fabry-Perot interferometric sensors," Opt. Lett. 17, 1021-1024 (1992).

2. A. Wang, S. Gollapudi, R. G. May, K. A. Murphy, and R. O. Claus, "Advances in sapphire-fiber-based interferometric sensors," Opt. Lett. 17, 1544-1546 (1992).

3. K. A. Murphy, M. F. Gunther, A. M. Vengsarkar, and R. O. Claus, "Quadrature phase-shifted, extrinsic Fabry-Perot optical fiber sensors," Opt. Lett. 16, 273-275 (1991).

4. K. A. Murphy, M. S. Miller, A. M. Vengsarkar, and R. O. Claus, "Elliptical-core, two-mode, optical fiber sensor implementation methods," J. Lightwave Technol. 8, 1688-1695 (1990).

5. J. A. Greene, K. A. Murphy, B. R. Fogg, R. O. Claus, and A. M. Vengsarkar, "Optical fiber, vibration mode filters incorporating photoinduced refractive index gratings," Smart Mater. Struct. 1, 243-249 (1992).

6. G. Keiser, Optical Fiber Communications, 2nd ed. (McGrawHill, New York, 1991).

7. W. B. Spillman, Jr. and D. H. McMahon, "Frustrated total internal reflection multimode fiber optic hydrophone," Appl. Opt. 19, 113-117 (1980).

8. A. W. Palmer, J. R. Croft, and R. Valsler, "Potential applications for total internal reflection fibre optic sensors," in Fiber Optics '83 (London), L. R. Baker, ed., Proc. SPIE 374, 206-207 (1983).

9. K. T. V. Grattan, A. W. Palmer, and D. P. S. Saini, "Frustratedtotal-internal-reflection fiber-optic pressure sensor," J. Lightwave Technol. LT3, 1130-1134 (1985).

10. J. R. Hull, "Proposed frustrated total internal reflection acoustic sensing method," Appl. Opt. 20, 3594-3599 (1981).

11. D. E. Altman, "Frustrated total internal reflection fiber optic small-motion sensor for hydrophone use," U.S. patent 4,286,468 (Sept. 1981).

12. J. D. Beasly, "Evanescent fiber optic pressure sensor apparatus," U.S. patent 4,360,247 (Nov. 1982).

13. L. M. Brekhovskikh, Waves in Layered Media (Academic, New York, 1960).

14. K. Wilner and N. P. Murarka, "Design considerations and test results of an evanescent switch-attenuator," Appl. Opt. 20, 3600-3605 (1981). 